\title{
THE GEMOLOGICAL PROPERTIES OF CHATHAM FLUX-GROWN SYNTHETIC ORANGE SAPPHIRE AND SYNTHETIC BLUE SAPPHIRE
}

By Robert E. Kane

Recent rumors in the trade and inquiries to the various offices of GIA's Gem Trade Laboratory, Inc., concerning the commercial availability of faceted fluxgrown synthetic blue sapphires prompted the writing of this article. Blue as well as orange flux-grown synthetic sapphires are now commercially available from Chatham Created Gems, Inc, in limited quantities as rough crystal groups and single crystals; they have not yet been marketed as faceted gems. In this article, the author examines the gemological properties of Chatham flux-grown synthetic orange sapphires and blue sapphires.

\section{ABOUT THE AUTHOR}

Mr. Kane is gem identification supervisor of GlA's Gem Trade Laboratory, Inc., Los Angeles, California.

Acknowledgments: The author wishes to thank Thomas Chatham, president of Chatham Created Gems, Inc., for helptul discussions and the loan of most of the synthetic material used in this study; Michael Waitzman for useful suggestions; Joe Graf and Michael Clary, of the Los Angeles Gem Trade Laboratory, for obtaining the hydrostatic specific gravities; Tino Hammid for the photographs in figures 1,2, and 19, and Peter Johnston for the illustrations in figures 3, 4, 5 , and 6. All other photographs are by the author.

1982 Gemological Institute of America
7 he synthesis of transparent corundum in virtually been accomplished by the Verneuil technique (also known as the flame-fusion process) and successfully marketed since the early 1900s (Nassau, 1982). Synthetic ruby manufactured by other methods, including the flux process, has also been commercially available for many years now. The synthesis of flux-grown blue sapphire, however, was first accomplished only eight years ago, by Chatham Created Gems, Inc., and has not yet reached the same level of sophistication as flux-grown synthetic ruby. Because of problems with synthesis, flux-grown blue sapphire to date has been marketed in the trade only on a small scale, in the form of rough crystal groups and single crystals. Flux-grown blue sapphires have not been sold as faceted gemstones by the Chatham firm (Thomas Chatham, personal communication, 1982), although a few stones reportedly have been cut by purchasers of the rough and may be seen, though very rarely, by the gemologist.

Recently, Chatham was successful in synthesizing orange sapphire by the flux method. This material, too, is being sold only as crystal groups or as an occasional single crystal (Thomas Chatham, personal communication, 1982). Although we know of the synthesis of fluxgrown orange sapphire, and other colors, by J. P. Remeika in the early 1960s (Nassau, personal communication, 1982), these samples were grown for industrial use only.

The purpose of this article is to present the gemological properties of the Chatham flux-grown synthetic orange and synthetic blue sapphires, as well as means of distinguishing these synthetics from their natural counterparts. The author's intent is to provide the gemologist with the information necessary to conclusively identify flux-grown synthetic orange and synthetic blue sapphires should they become widely available commercially as cut 


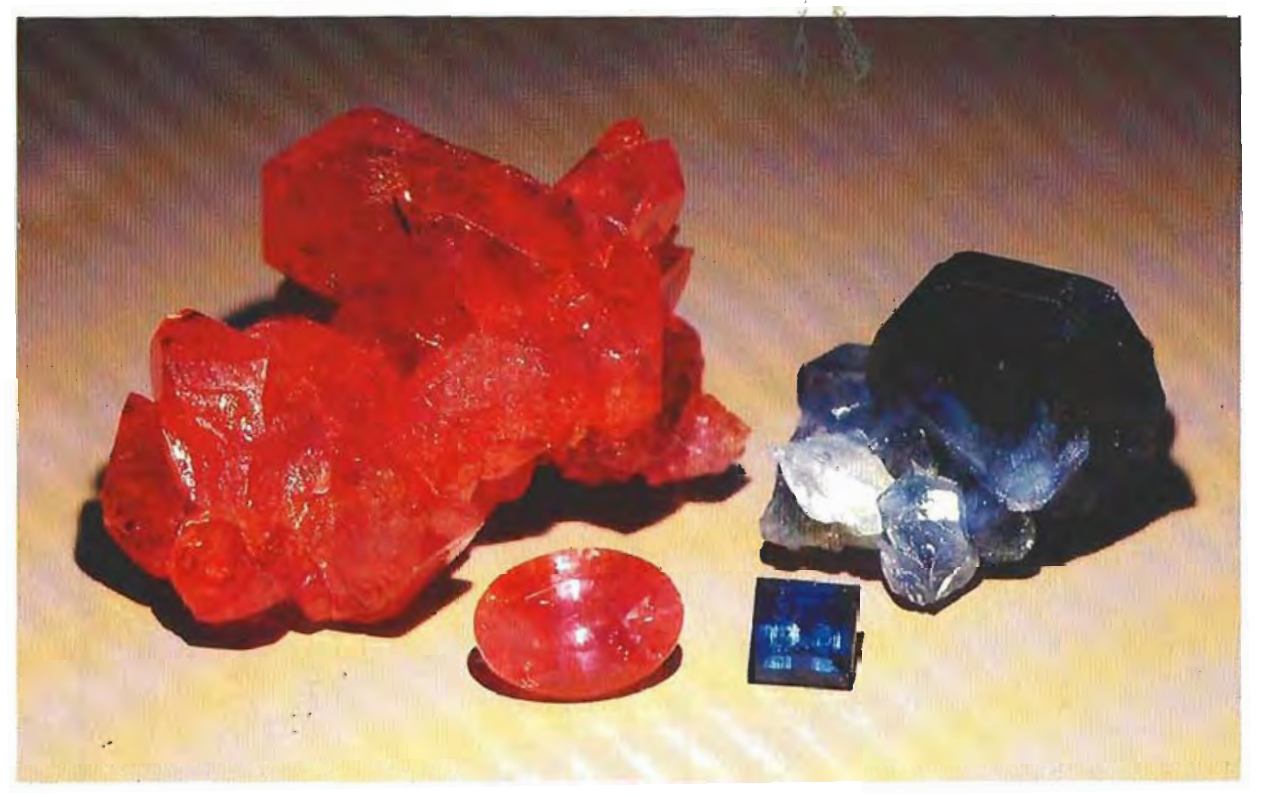

Figure 1. Exceptional examples of Chatham flux-grown synthetic orange sapphire (rough crystal group, $39.86 \mathrm{ct}$; faceted stone, $1,32 \mathrm{ct}$ ) and flux-grown synthetic blue sapphire (rough crystal group, $11.59 \mathrm{ct}$; faceted stone, $0.39 \mathrm{ct}$ ).

stones. The author conducted this study with 31 Chatham flux-grown orange sapphire crystal groups and single crystals (ranging in weight from 3 to $40 \mathrm{ct}$ ) and with 18 Chatham flux-grown blue sapphire crystal groups and single crystals (ranging in weight from 7 to $55 \mathrm{ct}$ ), several hundred flux-grown blue sapphire single crystals (weighing from 1 to 2 ct each), and 11 faceted flux-grown blue sapphires (weighing from 0.37 to $2 \mathrm{ct}$ each). Sample material is illustrated in figure 1, including a Chatham flux orange sapphire faceted by Bill Kerr of GIA for this study.

\section{DIFFICULTIES OF SYNTHESIZING FLUX-GROWN ORANGE SAPPHIRE AND BLUE SAPPHIRE}

Even though Chatham has not perfected the synthesis of blue sapphire by the flux method after nearly eight years of working on this project, they have had much greater success with the synthetic blue sapphire than they did with flux-grown ruby in the early years of its synthesis (Thomas Chatham, personal communication, 1982). Several different manufacturers have commercially synthesized ruby by the flux technique since the late 1950s; however, many years of experimentation were required to bring synthetic flux ruby to the state that we know it today.

The major difficulties encountered in the manufacture of the orange and blue varieties of flux-grown synthetic corundum derive from the fact that addition of slight amounts of the coloring agent impurities needed to create such colors causes disturbances in crystal growth. Each vari- ety has a unique set of problems. For example, the addition of iron and titanium to grow blue sapphire by the flux method causes extreme color zoning. Chatham states that they have attempted to alleviate color zoning by trying to control the speed and direction of crystal growth, which is directly related to the clarity of the material. This often results in many of the crystals being heavily included. At this time, Chatham has not solved all of the problems with the synthesis of flux blue sapphire. They currently are concentrating their efforts on eliminating the disruptions in the crystal structure to improve clarity.

Chatham states that they first synthesized orange sapphire by the flux method in 1981. As with the synthetic blue sapphire, Chatham presently sells only limited quantities of the orange material and only as rough crystal groups.

\section{GEMOLOGICAL CHARACTERISTICS OF FLUX-GROWN SYNTHETIC ORANGE SAPPHIRE AND BLUE SAPPHIRE}

Even though Chatham has been experimenting with the flux growth of synthetic blue sapphire since 1974, very little descriptive information concerning this synthetic has been published in the gemological literature, with the exception of some brief observations presented by Scarratt (1977) and Koivula (1981a). The following discussion examines in detail the gemological characteristics of the Chatham flux-grown orange sapphires and blue sapphires. These characteristics, many of which are the same for both colors, are summarized in table 1 . 
TABLE 1. The gemological properties of Chatham flux-grown synthetic orange sapphires and synthetic blue sapphires.

\begin{tabular}{|c|c|c|c|c|c|c|c|c|}
\hline \multirow{2}{*}{$\begin{array}{l}\text { Chatham } \\
\text { flux-grown } \\
\text { synthetic } \\
\text { sapphire }\end{array}$} & \multirow{2}{*}{$\begin{array}{l}\text { Refractive } \\
\text { index and } \\
\text { birefringence }\end{array}$} & \multirow[b]{2}{*}{ Pleochroism } & \multicolumn{3}{|c|}{ Luminescence } & \multirow[b]{2}{*}{$\begin{array}{l}\text { Absorption } \\
\text { spectrum }\end{array}$} & \multirow[b]{2}{*}{$\begin{array}{l}\text { Specific } \\
\text { gravity }\end{array}$} & \multirow[b]{2}{*}{ Inclusions } \\
\hline & & & $\begin{array}{c}\text { Long-wave U.V. } \\
\text { radiation }\end{array}$ & $\begin{array}{c}\text { Short-wave U.V. } \\
\text { radiation }\end{array}$ & $\mathrm{X}$-rays & & & \\
\hline Orange & $\begin{array}{c}1.762-1.770 \\
0.008\end{array}$ & $\begin{array}{l}\text { Strong pink- } \\
\text { orange and } \\
\text { brownish } \\
\text { yellow. }\end{array}$ & $\begin{array}{l}\text { Variable: intensity } \\
\text { ranges from } \\
\text { strong to very } \\
\text { strong; overall flu- } \\
\text { orescent color } \\
\text { ranges from or- } \\
\text { angy red through } \\
\text { reddish orange to } \\
\text { yellowish orange, } \\
\text { with zones of } \\
\text { chalky yellow. }\end{array}$ & $\begin{array}{l}\text { Intensity ranges } \\
\text { from very weak to } \\
\text { weak; same flu- } \\
\text { orescent colors as } \\
\text { long-wave. }\end{array}$ & $\begin{array}{l}\text { Variable: in- } \\
\text { tensity ranges } \\
\text { from strong to } \\
\text { very strong in } \\
\text { most cases, } \\
\text { with some } \\
\text { areas being in- } \\
\text { ert to very } \\
\text { weak; overall } \\
\text { fluorescent } \\
\text { color ranges } \\
\text { from reddish } \\
\text { orange to } \\
\text { orange, may } \\
\text { exhibit zoned } \\
\text { areas of chalky } \\
\text { yellow. No } \\
\text { phospho- } \\
\text { rescence. }\end{array}$ & $\begin{array}{l}\text { Absorption } \\
\text { lines at } 475, \\
476.5,468.5, \\
659.2,668, \\
692.8 \text { and } \\
694.2 \mathrm{~nm} \text {, and } \\
\text { broad absorp- } \\
\text { tion blocking } \\
\text { out all of the } \\
\text { violet and } \\
\text { some of the } \\
\text { blue, all of the } \\
\text { green and yel- } \\
\text { low, and a } \\
\text { small area in } \\
\text { the orange } \\
\text { portion of the } \\
\text { visible spec- } \\
\text { trum. Not } \\
\text { diagnostic. }\end{array}$ & $4.00 \pm 0.003^{a}$ & $\begin{array}{l}\text { Various forms of } \\
\text { flux; platinum; } \\
\text { dense, white, cloud- } \\
\text { like areas; } \\
\text { transparent crystals; } \\
\text { fractures; healed } \\
\text { fractures; color } \\
\text { zoning. }\end{array}$ \\
\hline Blue & $\begin{array}{c}1.762-1.770 \\
0.008\end{array}$ & $\begin{array}{l}\text { Strong violet- } \\
\text { ish blue and } \\
\text { greenish } \\
\text { blue. }\end{array}$ & $\begin{array}{l}\text { Variable: uneven } \\
\text { reaction, inert to } \\
\text { very strong; flu- } \\
\text { orescent colors } \\
\text { patchy, ranging } \\
\text { from chalky } \\
\text { greenish yellow to } \\
\text { chalky reddish } \\
\text { orange to yellow- } \\
\text { ish-brownish } \\
\text { green to sulfur } \\
\text { yellow. }\end{array}$ & $\begin{array}{l}\text { Variable: uneven } \\
\text { reaction, inert to } \\
\text { strong. The fol- } \\
\text { lowing fluores- } \\
\text { cent colors may } \\
\text { be observed: } \\
\text { chalky greenish } \\
\text { yellow, dull yel- } \\
\text { lowish green, dull } \\
\text { chalky greenish } \\
\text { white, chalky red- } \\
\text { dish orange, and } \\
\text { strong yellow. }\end{array}$ & $\begin{array}{l}\text { Variable: un- } \\
\text { even reaction, } \\
\text { inert to mod- } \\
\text { erate; overall } \\
\text { fluorescent } \\
\text { color fairly } \\
\text { consistent } \\
\text { chalky yellow- } \\
\text { ish white. No } \\
\text { phospho- } \\
\text { rescence. }\end{array}$ & $\begin{array}{l}\text { Weak diffused } \\
\text { band centered } \\
\text { at } 451.5 \mathrm{~nm} \text {. } \\
\text { Not diagnostic. }\end{array}$ & $4.00 \pm 0.03^{a}$ & $\begin{array}{l}\text { Various forms of } \\
\text { flux; platinum; } \\
\text { dense, white, cloud- } \\
\text { like areas; } \\
\text { transparent crystals; } \\
\text { fractures; healed } \\
\text { fractures; various } \\
\text { forms of color } \\
\text { zoning; thin, white- } \\
\text { appearing needles. }\end{array}$ \\
\hline
\end{tabular}

${ }^{a}$ Crystal groups with applied ceramic glaze are often lower, near 3.85. Platinum inclusions in crystals without applied ceramic glaze may raise S.G. 
Visual Appearance. As the Chatham synthetic sapphire crystals are examined with the unaided eye, several observations can be made on both varieties. Color, transparency, and clarity are the most obvious properties noted, although other unusual characteristics also come to light.

With regard to color, the overall hue of the flux-grown orange sapphires varies from orange to reddish orange in moderate to vivid saturation. Color zoning is often seen within the individual crystal groups and single crystals. There are areas of yellowish orange, orangy yellow, pinkish orange, and orangy pink.

Strong color zoning is evident in nearly all of the flux-grown blue sapphire crystals. The zones range from near colorless through light blue and medium blue to extremely dark blue (almost black). Many of the crystals have heavily included areas that appear white to the unaided eye. In some of the crystal groups, some small crystals will be nearly colorless and others will be zoned with areas that are near colorless or differing shades of blue.

With regard to transparency and clarity, both the orange and the blue flux-grown synthetic sapphires range from transparent to translucent, often within the same crystal. Some blue crystals were opaque. The translucency in both is due to the many areas that are heavily included. The opacity in the blue crystals results from the dark, almost black, color. The clarity to the unaided eye ranges from areas that appear to be free from inclusions (except color zoning) to areas that are very heavily included.

Further examination with the unaided eye reveals a transparent, near-colorless glossy coating on the backs of many of the crystal groups. This is most evident at crystal junctures, where the coating has accumulated. When a pen light is used for horizontal illumination, gas bubbles may be observed in the coating.

Microscopic examination confirms the presence of a coating and the occurrence of many spherical gas bubbles at some of the crystal junctions (figure 2). Chatham states that a liquid silica-based ceramic glaze is applied to the backs of the crystal groups and the crystals fired in a kiln at $1000^{\circ} \mathrm{C}$. This practice was started several years ago on some synthetic ruby crystal groups to strengthen those that were thin and fragile. Chatham now routinely applies a ceramic glaze to all of the synthetic orange and blue sapphire

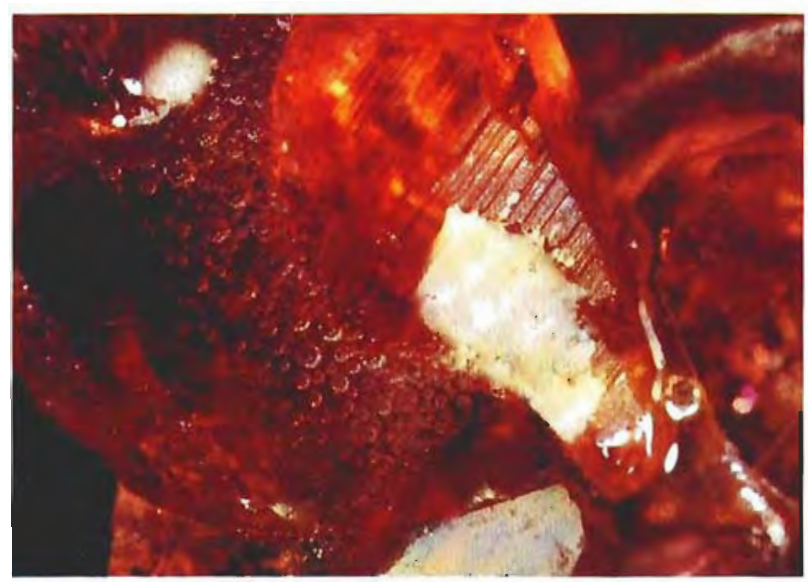

Figure 2. Dense concentration of spherical gas bubbles in the silica-based ceramic glaze applied to the back of this Chatham fluxgrown synthetic orange sapphire crystal group. Oblique illumination accentuates the termination of the glaze near the edge of the crystal group. Magnified $10 \times$.

and synthetic ruby crystal groups that they market. Chatham does not, however, put this ceramic glaze on single crystals (Thomas Chatham, personal communication, 1982).

Further examination with the microscope confirms that the ceramic glaze is found mostly on the backs of the crystal groups; occasionally, however, it is seen on the sides. The glaze often seeps between crystal faces and into surface fractures, where spherical and elongated flat gas bubbles may form. These bubbles may look like inclusions within the crystal, but they actually occur only between crystal faces or in fractures. In some of the thinner crystal groups, such gas bubbles in the back are visible from the front. The transparent glossy coating appeared to be essentially the same on both the orange and the blue crystal groups, with the exception that some of the synthetic blue sapphire crystal groups had glazed areas with an opaque, white, burned appearance. This is in contrast to the relatively consistent, transparent, near-colorless appearance of the coating on the synthetic orange crystal groups.

Refractive Indices and Birefringence. Refractive indices were obtained using a GEM Duplex II refractometer in conjunction with a sodium light source. In preparation for testing, one of the fluxgrown orange single crystals was faceted. This faceted stone and the faceted synthetic blue sap- 
phires were determined to be uniaxial negative with a refractive index of $\omega=1.762$ and $\epsilon=1.770$, and a corresponding birefringence of 0.008 .

Pleochroism. A calcite dichroscope was used to determine this property. In the flux-grown orange sapphires, the dichroic effect was observed as strongly distinct colors of pink-orange and brownish yellow. The synthetic blue sapphires revealed dichroism in distinct colors of violetish blue and greenish blue. As would be expected, the dichroic colors of both the synthetic orange and synthetic blue sapphires varied depending on the color of the crystal and how much color zoning was present in the area being examined.

Luminescence. Exposure to long-wave ultraviolet radiation of the 31 flux-grown synthetic orange sapphire crystals studied revealed variable fluorescence, from strong to very strong. The overall color of the fluorescence ranged from orangy red (almost pure red) through reddish orange to yellowish orange. All of the crystals had an opaque, dull appearance. Most of the crystals had zones of moderate to strong chalky yellow fluorescence. The crystals that were predominantly orange seemed to have more yellow fluorescent zoning than did the crystals with redder hues.

The fluorescent chalky yellow zones were observed on both sides of the flux-grown orange sapphire single crystals and crystal groups. The ceramic glaze on the crystal groups did not fluoresce and did not appear to affect the fluorescence in any way. These yellow zones were observed to range from being confined to one or two small areas to comprising almost $80 \%$ of the crystal.

Exposure of the flux-grown orange sapphires to short-wave ultraviolet radiation revealed essentially the same variable fluorescent reaction. The zoning and color of the short-wave ultraviolet fluorescence was the same as the long-wave fluorescence. The major difference was the intensity of the short-wave fluorescence, which ranged from very weak to weak.

Exposure of the flux-grown orange sapphires to X-rays also revealed a variable fluorescence. The intensity ranged from strong to very strong in most cases, with a few crystal groups exhibiting strong to very strong fluorescence around the edges and inert to weak centers. The overall color of the X-ray fluorescence ranged from reddish orange to orange. Some of the crystal groups and single crystals had zones of moderate chalky yellow X-ray fluorescence that seemed to correspond to the chalky yellow zones of long-wave and shortwave ultraviolet fluorescence. No visible phosphorescence was observed after X-ray excitation.

Almost all of the flux-grown synthetic blue sapphires exhibited a very patchy, uneven reaction to long-wave ultraviolet radiation. Some areas were inert, while others ranged in intensity from very weak to very strong.

In the material examined by the author, many of the extremely dark blue areas were inert; the moderately dark blue areas exhibited a very weak, dull, yellowish-brownish green; the medium-blue to near-colorless areas glowed a weak to strong greenish yellow; the colorless and heavily fluxincluded areas fluoresced the same greenish yellow, but much more so than the other areas; some of the clean, near-colorless areas fluoresced a weak chalky reddish orange; and the whitish, burned (coated) areas fluoresced a very strong sulfur yellow. Because of possible optical irregularities and unobserved inclusion centers, as well as the obstruction of some areas within the crystal groups, these observations of corresponding areas of color and fluorescent reactions cannot be considered conclusive at this time, but may be viewed as good indications of the synthetic nature of the material.

Exposure of the synthetic blue material to short-wave ultraviolet radiation also revealed a very patchy, uneven reaction. Some areas were inert, while others ranged in intensity from very weak to strong. The color of the fluorescence varied widely, with the following hues observed: chalky greenish yellow; dull yellowish green; dull, chalky, greenish white; chalky reddish orange; strong yellow; and chalky whitish blue (observed in several small, near-colorless fragments).

Exposure of the flux-grown synthetic blue sapphires to X-rays also revealed a patchy reaction. Some areas were inert, and others ranged in intensity from very weak to moderate. The X-ray fluorescence was a fairly consistent chalky yellowish white. There was no visible phosphorescence after exposure to X-rays.

As Webster noted (1975), some natural blue sapphires will change to a "dirty amber colour" when exposed to X-rays. This color change is not permanent; the original color returns after about $3 \frac{1}{2}$ hours' exposure to sunlight or even much more rapidly when the stone is heated to a tem- 


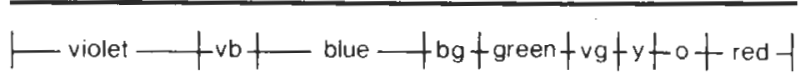

A

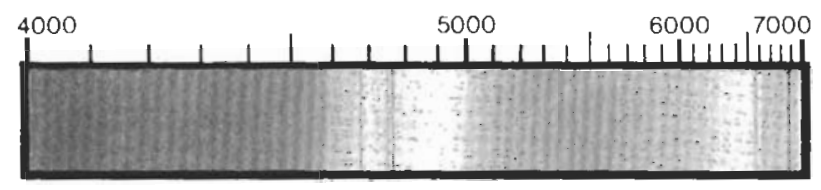

B

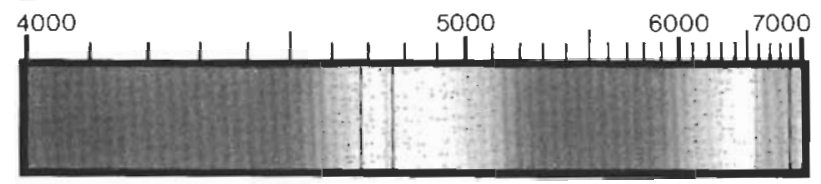

Figure 3. Drawings of absorption spectra for (A) Chatham flux-grown synthetic orange sapphire and $(B)$ Chatham flux-grown synthetic ruby, as observed on a direct-vision spectroscope (in $\AA$ ) at room temperature.

perature of about $230^{\circ} \mathrm{C}$. Scarratt (1977) reported that when a few Chatham flux-grown blue sapphire crystals were exposed to X-rays, "the colorless and some of the pale blue areas photocoloured to varying depths of green or yellow, depending on the length of exposure." He also indicated that the induced colors were not permanent. Unfortunately, neither Webster nor Scarratt stated the length of exposure time required for the $\mathrm{X}$-rays to produce such a color change. The Chatham synthetic blue sapphires examined by the author displayed no change in color after exposure to X-rays for 5 to 10 seconds.

Absorption Spectra. The visible light absorption spectra of the 31 synthetic orange sapphire crystals were examined with the GEM spectroscope unit. The observed spectra exhibited essentially the same transmission and absorption features as the diagnostic absorption spectrum described by Liddicoat (1981) for natural and synthetic ruby, purple sapphire, and dark "padparadscha" sapphire. The absorption features, however, are much weaker in the Chatham product. Figure 3 illustrates the absorption spectra for (A) Chatham fluxgrown orange sapphire and (B) Chatham fluxgrown ruby as observed in the GEM spectroscope at room temperature.

The characteristic spectrum for the Chatham flux-grown orange sapphire has two major transmission areas, one in the blue and one in the red. Within the blue transmission area are three sharp narrow lines: one at $468.5 \mathrm{~nm}$ and a very close doublet at 475 and $476.5 \mathrm{~nm}$. The line at $475 \mathrm{~nm}$ is extremely faint and difficult to observe. In the

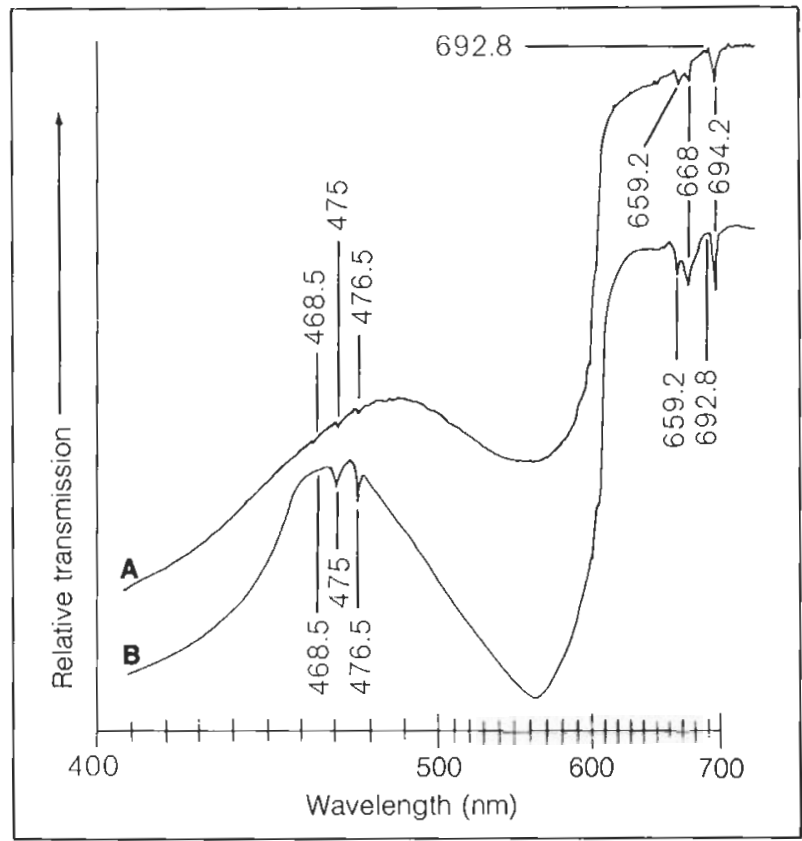

Figure 4. Visible-light spectral transmission curves for (A) Chatham flux-grown synthetic orange sapphire and (B) Chatham flux-grown synthetic ruby, as documented by the automatic recording spectrophotometer at $60 \mathrm{~K}$.

red portion of the visible spectrum is the typical chromium absorption that is often associated with corundum. There are very faint, narrow lines at $659.2 \mathrm{~nm}$ and $668 \mathrm{~nm}$, along with two stronger, yet narrow, lines very closely spaced at $692.8 \mathrm{~nm}$ and $694.2 \mathrm{~nm}$.

In addition to these areas, there is a broad absorption blocking out all of the violet and some of the blue, all of the green and yellow, and a small area in the orange portion of the visible light spectrum. All of the synthetic orange sapphires exhibited this absorption spectrum. The thicker specimens exhibited a stronger spectrum than did the thinner crystals.

Using a modified Zeiss $\mathrm{PMQ}_{3}$ recording spectrophotometer, Stephen Hofer, of GIA's Department of Research, confirmed this absorption spectrum. Figure 4 shows the visible-light spectral transmission curves for the Chatham fluxgrown orange sapphire (A) and the flux-grown ruby $(\mathrm{B})$, as documented by the automatic recording spectrophotometer.

The absence or presence of this spectrum cannot be considered diagnostic at this time. Although orange sapphires are quite rare in nature, when they occur in the same color as their Chatham synthetic counterpart, they may exhibit an absorption spectrum very similar if not iden- 


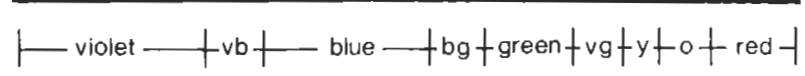

A

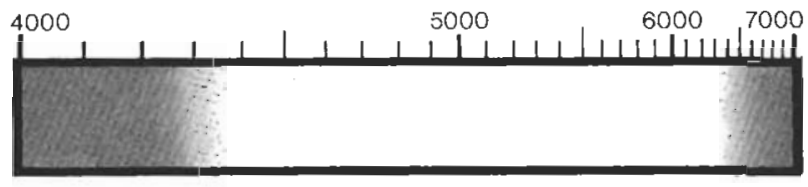

\section{B}

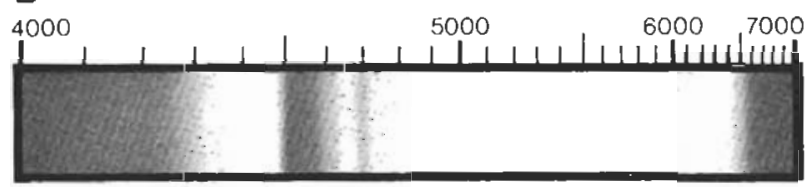

C

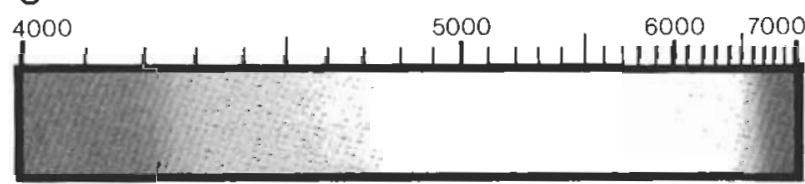

Figure 5. Drawings of absorption spectra for $(A)$

Chatham flux-grown synthetic blue sapphire,

(B) natural dark blue sapphire, and (C)

Verneuil synthetic blue sapphire, as observed

on a direct-vision spectroscope (in $\AA$ ) at room

temperature. Absorption spectrum A may be

observed in some natural medium blue

sapphires, and absorption spectrum $C$ may be

observed in light blue unheated natural

sapphires as well as in medium blue heat-

treated natural sapphires.

tical to the one that is characteristic of the Chatham product.

However, they may also show distinct differences. The natural color of orange sapphires is often attributed to a combination of iron and chromium, both of which are also found in the Chatham synthetic orange sapphire. If iron only appears in the spectrum, it may be observed in bands centered near 450, 460, and $470 \mathrm{~nm}$ (Liddicoat, 1981) that will not appear in the Chatham synthetic. Natural orange sapphires may also show chromium bands only, or perhaps no perceivable absorption features at all. These natural, unheated sapphires are often brownish orange, yellowish orange, or orange, and not reddish orange. To complicate matters even further, an orange color that may be slightly different loften brownish orange or yellowish orangel from the color of the Chatham flux-grown sapphire may be produced in natural sapphires by heat treatment or irradiation (Crowningshield and Nassau, 1981). These treated stones often will exhibit a featureless visible-light absorption spectrum.

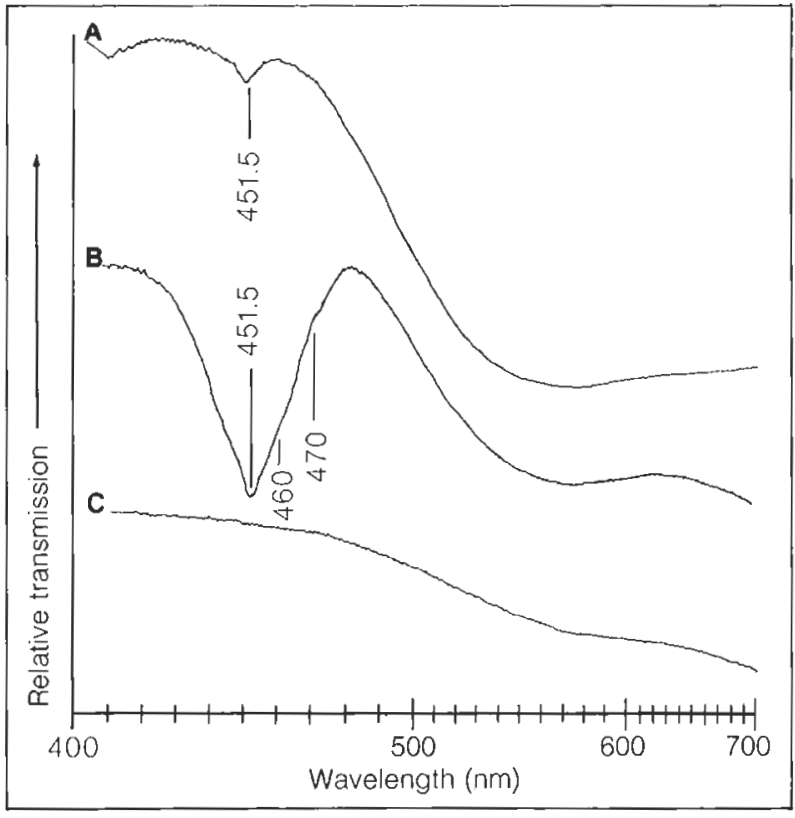

Figure 6. Visible-light spectral transmission curves for (A) Chatham flux-grown synthetic blue sapphire, (B) natural dark blue sapphire, and (C) Verneuil synthetic blue sapphire, as documented by the automatic recording spectrophotometer at $60 \mathrm{~K}$. Some natural medium blue sapphires exhibit the same visible-light spectral transmission curves as $A$, and some light blue unheated natural sapphires and medium blue heat-treated natural sapphires may exhibit a lack of absorption features as in $C$; the curve, however, may vary.

Spectrographic examination of the visible-light spectra of the Chatham flux-grown blue sapphires also was performed with the GEM spectroscope unit. Most of the stones showed a very faint diffused band slightly above $450 \mathrm{~nm}$. The band was quite vague and difficult to see in many of the stones. Figure 5 compares the absorption spectra for (A) Chatham synthetic blue sapphire, (B) natural dark blue sapphire, and (C) Verneuil synthetic blue sapphire, as observed on the GEM spectroscope unit at room temperature. Again using the modified Zeiss $\mathrm{PMQ}_{3}$ recording spectrophotometer, Hofer confirmed the presence of this absorption band and showed it to be centered at $451.5 \mathrm{~nm}$ (figure 6).

The presence or absence of the $450 \mathrm{~nm}$ band can no longer be considered diagnostic in and of itself. For many years it was accepted that virtually all natural blue sapphires exhibit an absorption band centered at $450 \mathrm{~nm}$ that might be accompanied by two additional weaker bands 
centered near 460 and $470 \mathrm{~nm}$ (Anderson and Payne, 1955). However, the absorption bands in the blue portion of the visible spectrum in natural blue sapphire are due to iron in the ferric state; these bands decrease considerably in intensity with the decrease in iron content (Webster, 1975). Accordingly, there are natural sapphires that owe their color to titanium and iron that will not show any $450 \mathrm{~nm}$ band when examined with a standard hand-held type of spectroscope. There are also heat-treated blue natural sapphires, quite prevalent in today's market (Abraham, 1982), that will not exhibit the $450 \mathrm{~nm}$ band (Crowningshield and Nassau, 1981; Nassau, 1981).

However, the combination of the 450, 460, and $470 \mathrm{~nm}$ bands, commonly known as the 450 complex (Webster, 1975), has not been observed in any synthetic blue sapphires as of yet and can still be considered diagnostic of natural blue sapphires.

Specific Gravity. The specific gravity values for both the orange and the blue flux-grown synthetic sapphires were determined by means of the hydrostatic technique, using a Voland diamond balance along with the necessary specific gravity attachments. As would be expected, the applied ceramic glaze affected the specific gravity values. The lower specific gravity of the ceramic glaze, which was determined to be $3.088^{*}$, lowered the values of some of the glazed crystals from the expected values for corundum. The specific gravity of the flux-grown orange crystals with the applied ceramic glaze varied considerably, from 3.865 to 3.994. Several single crystals of flux-grown orange sapphire, which did not have the applied ceramic glaze, showed minor variations in density between 3.986 and 4.032 (within the accepted values for corundum).

The synthetic blue sapphire crystal groups that had the applied glaze ranged in value from 3.848 to 3.987 . Faceted material and single crystals that did not have the applied glaze showed minor variations in density from 3.974 to 4.055 . Platinum inclusions may increase slightly the specific gravity of flux-grown synthetic corundum.

- The presence of a large number of gas bubbles in the glaze will undoubtedly lower the specific gravity of this material.
Inclusions. As with essentially all other synthetic corundum, especially synthetic ruby, the most important means of identifying flux-grown synthetic orange sapphires and blue sapphires is their characteristic inclusions. Microscopic examination of the Chatham synthetic orange and synthetic blue sapphire crystals and faceted synthetic blue sapphires revealed a wide variety of inclusions. Most of these can be considered diagnostic of synthesis. However, some of the inclusions in the flux-grown blue sapphire-for example, very thin, white-appearing needles and straight and "hexagonal" color banding-could be quite confusing if used as the sole source of identification. If all of the inclusions observed, however, are carefully examined and considered in the identification, the Chatham flux-grown orange sapphires and blue sapphires should not present any great problems for the gemologist.

Many easily identifiable and diagnostic inclusions are characteristically found in the orange and blue Chatham synthetic sapphires. To date, the following inclusions have been observed in both: various forms of flux; platinum; dense, white, cloud-like areas; transparent crystals; fractures; healed fractures; and color zoning. Observed in the synthetic blue sapphire and not in the synthetic orange sapphire were thin, whiteappearing needles.

Flux, Residual, unmelted flux typical of fluxgrown synthetics was prevalent in nearly all of the Chatham flux-grown orange and blue sapphires examined. Several forms were observed. "Fingerprints" of flux that ranged from transparent and near colorless to opaque and white were seen in tightly arranged, thin, mesh-like patterns and loosely arranged, wide, flat, mesh-like patterns that frequently intersected one another (figure 7). The opaque, white "fingerprints" were usually observed in very high relief (figure 8). The transparent and near-colorless patterns were often observed in very low relief and may be more visible at certain viewing angles and lighting conditions than at others.

Most characteristic of the flux inclusions were white, wispy veils (figure 9), usually very fine in texture though occasionally moderately thick, that were seen in high relief. In many of the fluxgrown blue sapphire crystals, the wispy veils were in such densely concentrated areas that they ap- 


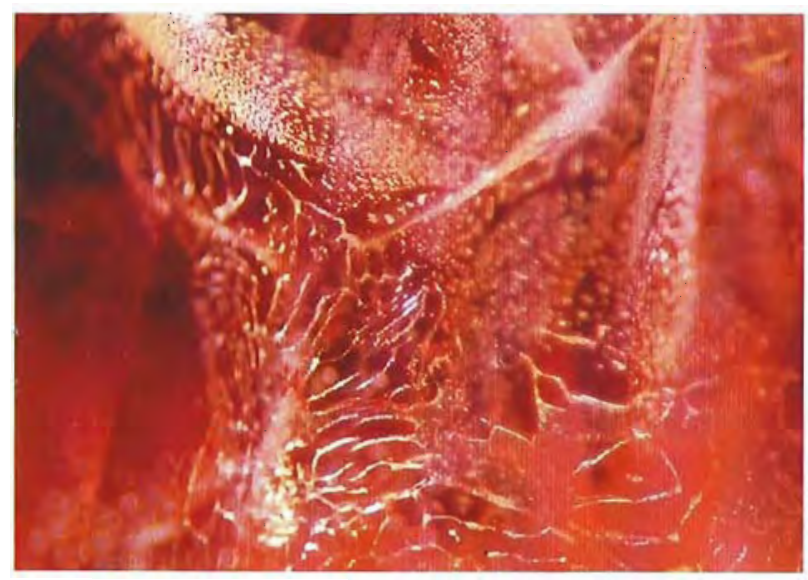

Figure 7. Transparent and nearly colorless, wide, mesh-like patterns of flux intersected by tightly arranged white "fingerprints" of flux in a Chatham flux-grown synthetic orange sapphire. Magnified $30 \times$.

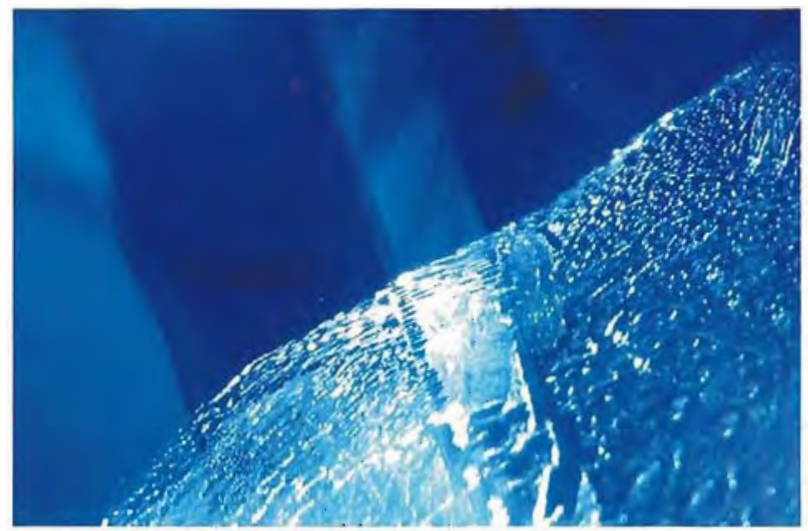

Figure 8. "Fingerprint" of white flux observed in high relief with straight-sided, angular, cloudy white zoning in a Chatham flux-grown blue sapphire. Magnified $25 \times$.

peared very white to the unaided eye. Opaque white veils that are not wispy in appearance, but are somewhat flat and curved, also were observed in both the synthetic orange and synthetic blue sapphires. They ranged from fine to moderate in texture. What has not been observed in any of the flux-grown orange and flux-grown blue sapphires are flux veils or "fingerprint" patterns that are as thick and globular as the very coarse flux "fingerprints" often thought to be typical of this type of synthesis and characteristically seen in some Kashan and Chatham synthetic rubies.

Another type of flux that was observed, although not as frequently as the ones discussed above, occurred as opaque globules that ranged from white to yellowish brown and from small to

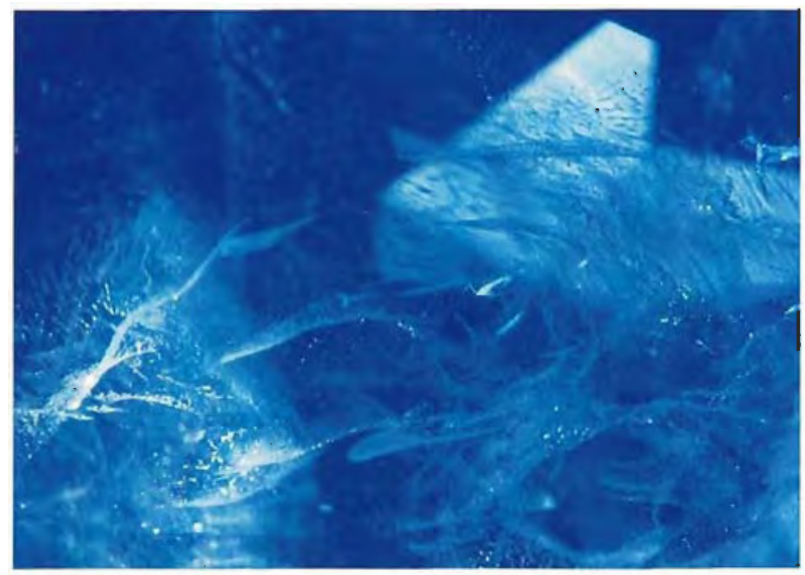

Figure 9. White, wispy veils of flux, fine in texture, betray the synthetic origin of this Chatham flux-grown blue sapphire. Note the thin, white-appearing needle in the upper left of this photomicrograph. Magnified $15 \times$.

extremely large (see figure 10). They were present as nondescript droplets or in somewhat angular forms with rounded corners. This type of inclusion was found to be more prevalent in the blue than in the orange material.

Platinum. Perhaps as characteristic as the several forms of flux inclusions is the presence of platinum. Chatham claims, as do other manufacturers of synthetic gemstones (Knischka and Gübelin, 1980) that they can control the formation of metallic inclusions during the growth process. The ability to repress or completely eliminate metallic inclusions is supported by the fact that many synthetics contain very few if any of these inclusions. For example, the Kashan synthetic rubies and pink sapphires contain virtually no platinum inclusions, although platinum inclusions were reported in some of the early material. However, platinum inclusions were quite prevalent in all 31 of the Chatham flux-grown orange sapphire crystals and in most of the Chatham flux-grown blue sapphire crystals and faceted stones that were examined. They are extremely diagnostic when present. Carol Stockton, of GIA's Department of Research, used the scanning electron microscope-energy dispersive spectrometer (SEM-EDS) system to perform chemical analyses on randomly selected metallic inclusions in both the Chatham flux-grown orange and flux-grown blue sapphire, and confirmed their identity as platinum.

Platinum inclusions may result from the flux process. For a substance to occur as an inclusion, the constituents of the inclusion must be present 


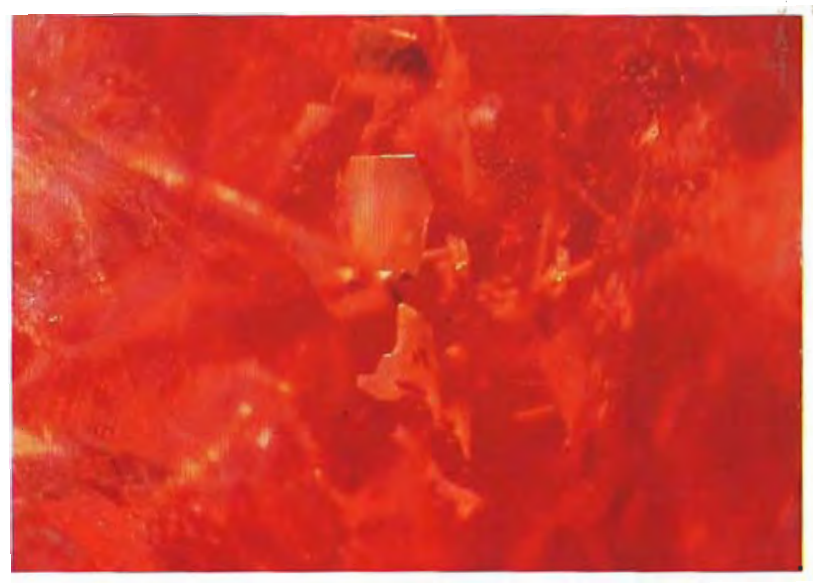

Figure 10. Opaque white globules of flux in a Chatham flux-grown synthetic orange sapphire. Magnified $20 \times$.

in the growth environment. With this type of synthesis, crystals are grown in a metallic, often platinum, crucible. Under the conditions necessary for flux growth, everything in the growth environment will go into solution, at least to some extent. Among the noble metals, platinum is probably the most nearly immune to this; under certain conditions, however, some of the platinum will go into the solution and may become part of the growing crystal.

In the Chatham synthetic orange sapphire and blue sapphire crystals, the platinum inclusions were observed to occur in several different forms: large, irregular flakes that ranged from thin to very thick, thin hexagonal and triangular platelets that occurred frequently in very symmetrical forms but sometimes in distorted forms, spikes, splinters, and small thin flat needles, as well as in other geometric and nondescript forms. These platinum inclusions range from very small to quite large (see figures 11 and 12).

Despite the diversity of forms in which the platinum occurs, this metallic inclusion provides the gemologist with a diagnostic and easily identifiable characteristic. Platinum inclusions occur only in synthetic gem materials and never in natural gemstones. When platinum occurs as an inclusion it is always opaque and may be black or shiny and metallic appearing (again, see figures 11 and 12). Differences in illumination and viewing angle often change the appearance from a shiny platinum color to black or vice versa.

In most of the synthetic orange sapphire crystals examined, the platinum inclusions were randomly oriented throughout, with the addition of a distinct "phantom" layer of growth near the

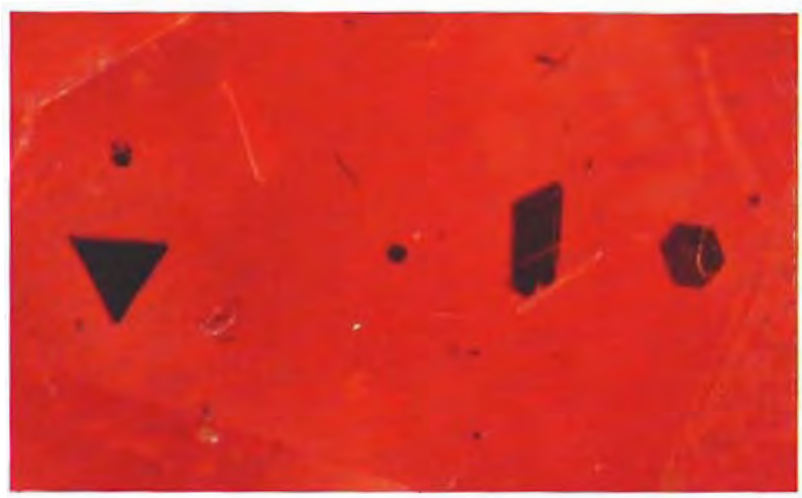

Figure 11. Very thin flakes of platinum included in a Chatham flux-grown synthetic orange sapphire. The platinum appears black because of the ultra-thin nature of the flakes and the lighting conditions. Slight changes in illumination often reveal the shiny metallic appearance usually associated with platinum. Magnified $60 \times$.

Figure 12. The shiny metallic appearance of platinum is very evident in this large, thick, angular platinum inclusion in a Chatham fluxgrown synthetic blue sapphire. Magnified $35 \times$.

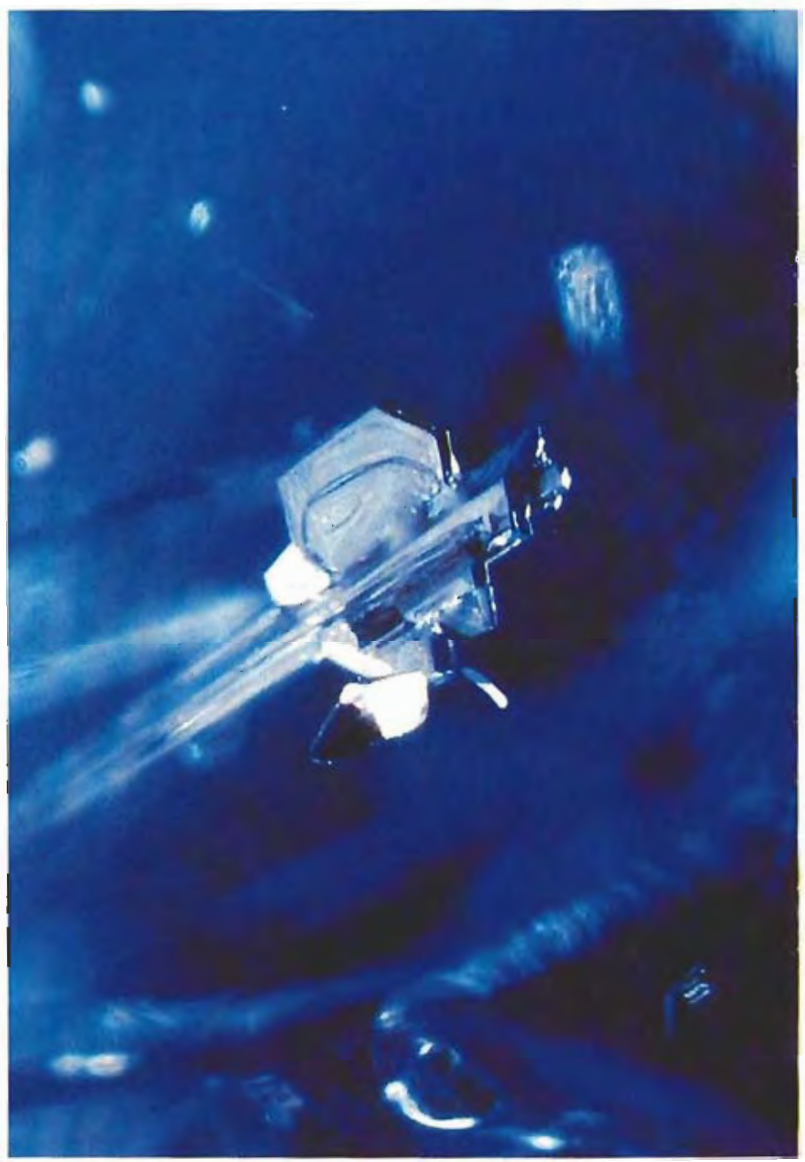




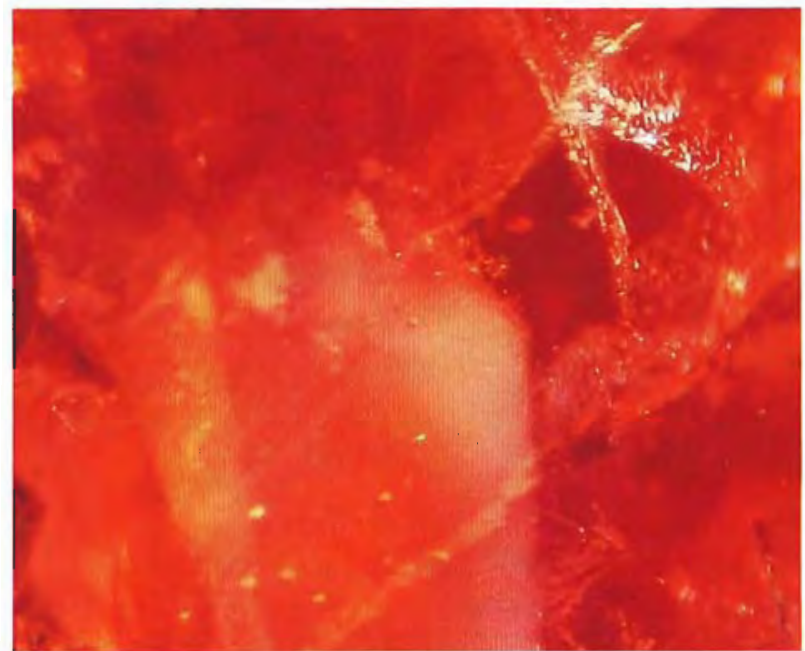

Figure 13. This cloud-like area in a Chatham flux-grown synthetic orange sapphire is composed of a dense concentration of minute, white, dust-like particles in association with larger forms of flux and bordered by pink color zoning, Magnified $30 \times$.

outer edges of many of the crystals. This layer of growth is seen easily with overhead illumination, and the boundaries of the growth layer are also readily apparent. Within this growth zone there is an excessive amount of platinum, much greater than is found anywhere else in the crystals. This would indicate a change in environment toward the end of the growth process. Although these thin growth zones would probably be cut away during faceting, this interesting characteristic was observed in many of the flux-grown orange sapphire crystals studied.

Such a layer of growth was also observed in some of the flux-grown blue sapphire crystals studied, but only very rarely; this, too, would probably be removed in faceting. The main difference between the platinum inclusions in the flux-grown orange sapphires and those in the fluxgrown blue sapphires is that the platinum in the synthetic orange sapphires examined was found to be relatively consistent in abundance in all of the crystals, whereas it varied considerably in the flux-grown blue sapphires studied. Specifically, some of the synthetic blue sapphires had virtually no platinum inclusions, others had only several extremely large platinum inclusions, and still others were moderately to very heavily included with platinum.

Dense, Cloud-Like Areas. Many of the Chatham synthetic crystals, both orange and blue, studied contained randomly oriented areas of very dense

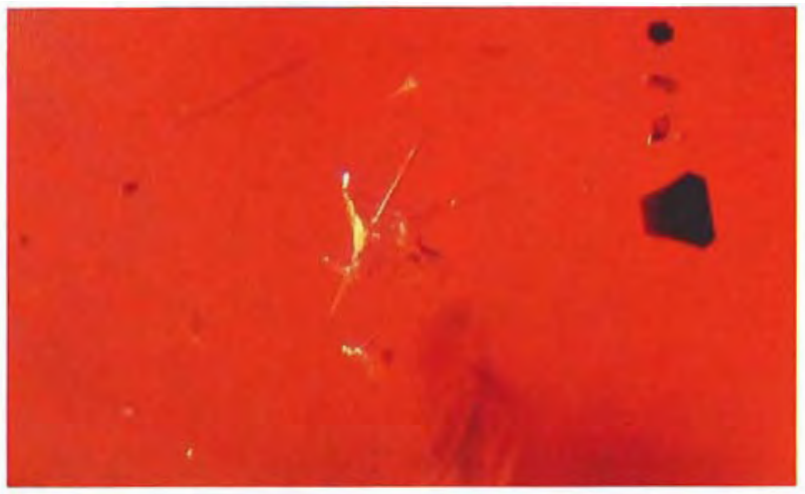

Figure 14. Transparent included crystal and ultra-thin flakes of platinum in a Chatham flux-grown synthetic orange sapphire. Magnified $70 \times$.

white clouds composed of minute, dust-like particles. The nature of the clouds was not resolvable at $120 \times$ magnification. Other inclusions, such as very small particles of platinum and globules of flux, were sometimes randomly oriented within these cloud-like areas (figure 13). The clouds in the Chatham flux-grown orange and blue sapphires are quite reminiscent of clouds observed in many natural blue sapphires, some Burmese rubies, some Chatham synthetic rubies, and some of the Knischka synthetic rubies (Knischka and Gübelin, 1980), as well as in other gemstones.

Transparent Crystals. Included in many of the synthetic orange sapphire crystals were transparent, near-colorless, "ghost-like" crystals, many of which were very small, typically ranging in length from $0.025 \mathrm{~mm}$ to $0.10 \mathrm{~mm}$, although some were much larger. Because of their very low relief, these small crystals often were very difficult to see with dark-field illumination and became easily visible only under certain lighting conditions, such as fiber-optic illumination, and with certain viewing angles. Consequently, these crystals are very difficult to photograph. Figure 14, however, does show one crystal that had a higher relief and a slightly different nature.

In some of the synthetic blue sapphires, transparent, near-colorless crystals were also observed. Some of the crystals are very similar to those observed in many of the flux-grown orange sapphires, in that they are low in relief and have a "ghost-like" nature. More commonly they are seen in a higher relief and are readily apparent. Some are hexagonal tabular crystals with very angular faces and easily visible straight and angular growth striations on several crystal faces /figure 


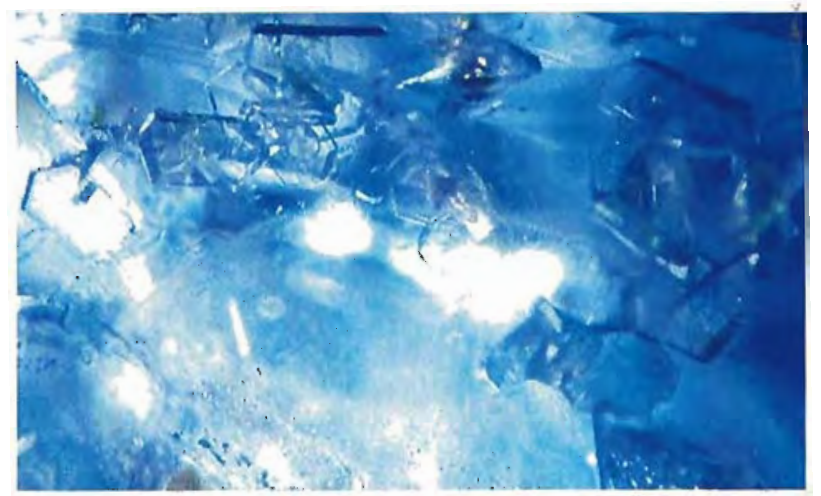

Figure 15. A cluster of transparent, colorless hexagonal tabular crystals included in a Chatham flux-grown synthetic blue sapphire. Magnified $35 \times$.

15). The crystals are observed to occur singly, in groups of several, or in clusters of a dozen or more.

The transparent crystals observed in both the synthetic orange and synthetic blue sapphires are very similar in nature, but not in shape, to transparent, near-colorless crystals seen in some Chatham flux-grown rubies (Kane, 1981). The crystals in both the orange and the blue Chatham synthetic sapphires are often tabular with rounded corners, although the crystals in the Chatham synthetic blue sapphires may be hexagonal and tabular with very sharp angular corners. In contrast, the crystals observed in some Chatham synthetic rubies are angular and not tabular, with rounded corners.

One of the transparent crystals included in the Chatham flux-grown blue sapphire shown in figure 15 reached the surface. X-ray diffraction analysis, performed by GIA's Chuck Fryer, revealed a pattern almost identical to that of chrysoberyl $\left(\mathrm{BeAl}_{2} \mathrm{O}_{4}\right)$. The lines in the pattern were the same as for chrysoberyl; however, the spacing was slightly different. Chatham states that when experimenting with the synthetic blue sapphires, they would often add many different elements and combinations of elements to the basic aluminum oxide formula $\left(\mathrm{Al}_{2} \mathrm{O}_{4}\right)$. These synthetic blue sapphires were part of a group to which they had added heavy concentrations of beryllium, which explains the presence of these inclusions. At the time this article is being written, further research is being undertaken to identify the small "ghost-like" crystals mentioned above.

Fractures. Several fractures and healed fractures were observed in the Chatham synthetic orange

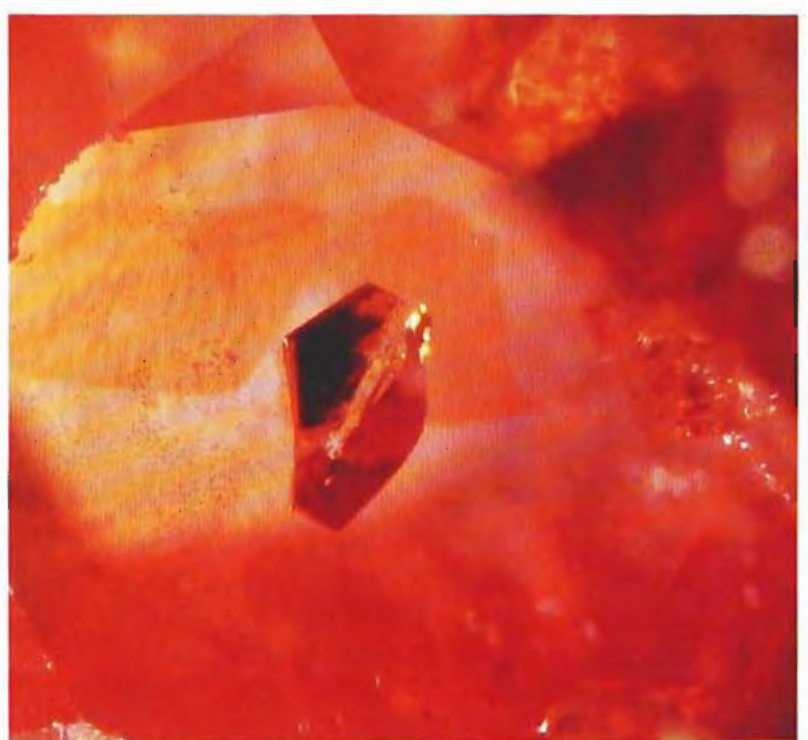

Figure 16. Yellow, orange, and pink irregular zoning borders a thick platinum inclusion in a Chatham flux-grown synthetic orange sapphire. Magnified 20x.

sapphire and blue sapphire crystals. They cannot, however, be considered diagnostic of synthesis, since they are similar in appearance to those seen in natural sapphire. Many of these fractures and healed fractures were iridescent and reflective at certain viewing angles when examined with either dark-field or oblique illumination.

In addition to those fractures observed within many of the flux-grown blue sapphire crystals and faceted stones, many fractures were found to occur between the crystals within a group. Many of these fractures were also very iridescent. The presence of these fractures between crystals, and the fact that crystal groups without a ceramic glaze were fragile and broke easily, indicates a poor cohesion.

Color Zoning. Color zoning of pink, orange, and yellow was observed in many of the crystal groups and single crystals of the flux-grown synthetic orange sapphires. Zoning occurred both confined to individual crystals within a group and in large sections of the crystal group. The same type of zoning may also be seen in single crystals. This color zoning may be straight or irregular; it does not appear to have a definite repetitious pattern (see figure 16).

Color zoning was also quite prevalent in nearly all of the Chatham flux-grown blue sapphire crystals and faceted stones that were examined. The color zoning was observed in a number of differ- 


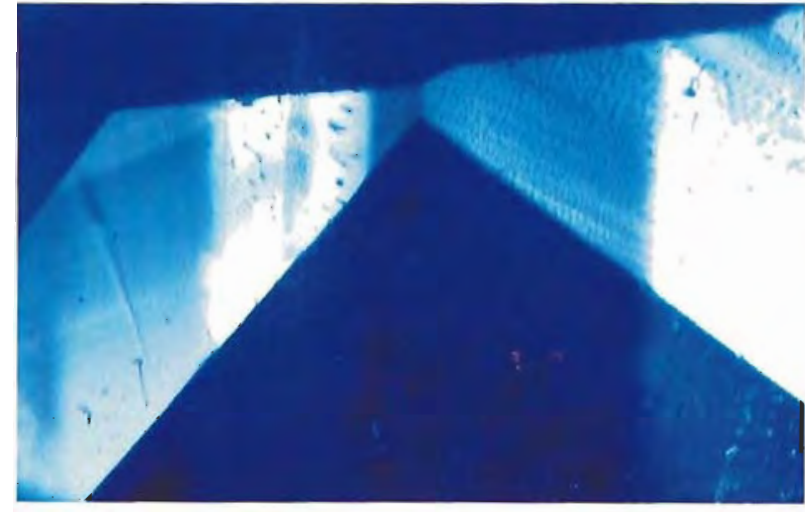

Figure 17. Straight-sided "hexagonal" zoning, colorless and blue, reflects the original growth and structure of this Chatham flux-grown synthetic blue sapphire. Magnified $35 \times$.

ent forms, some of which may be confusing to the gemologist. The zoning ranged from colorless to extremely dark blue. The following forms were noted: straight parallel zoning, straight-sided angular patches (figure 17), dark blue zoning arranged in "hexagonal" patterns with two to five sides / the sixth side of the hexagonal pattern was probably present in one crystal that was examined, but was obscured from view by an adjacent crystal), straight and "hexagonal" parallel cloudy white zoning (figure 18 and, again, figure 8), and irregular curved dark blue areas.

Some of the color-zoned areas reflected the growth and structure of the synthetic host crystal, while others appeared to be randomly oriented. Most of the zoning was easily visible with dark-field illumination. These zoned areas were even more readily apparent with the use of diffused illumination. A very simple method of obtaining diffused lighting is to place a piece of white tissue paper or a thin sheet of translucent white plastic between the stone and the light source, which can be dark-field or transmitted illumination. Diffused lighting also reveals areas of zoning that are not visible with dark-field illumination. Figure 19 shows the effect produced when flux-grown synthetic orange sapphire and blue sapphire are immersed in methylene iodide and viewed over diffused illumination.

Thin, White-Appearing Needles. Observed in some of the synthetic blue sapphires and not in the flux-grown orange sapphires, were extremely thin, white-appearing needles (figure 20). They may be somewhat short or very long, extending through most of the stone. They are usually observed singly or in groups of just a few, and were

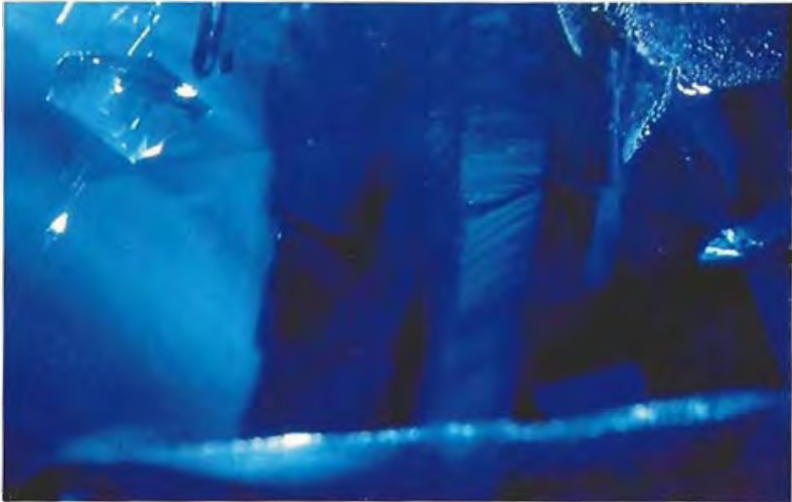

Figure 18. Straight and "hexagonal" parallel, cloudy white zoning in a Chatham flux-grown synthetic blue sapphire. Note the needle-like appearance of this zoning confined to one area in the upper center portion of this photomicrograph. Magnified $20 \times$.

not seen to intersect as they often do in natural blue sapphires. They could, however, very rarely intersect one another. Most likely, they would not be observed in the quantity that is often seen in natural blue sapphires.

These needles were observed not only alone but also as a gradual extension of platinum spikes and splinters. Even though the appearance of the needles was not reminiscent of platinum, the gradual extension off other platinum inclusions suggested that they were in fact platinum. One of the faceted synthetic blue sapphires contained an extremely thin, white-appearing needle that broke the surface. Chemical analysis performed by George Rossman and Randy Heuser, at the California Institute of Technology, on an SEM-EDS system confirmed that the needle was platinum.

Extremely thin, parallel, white-appearing needles were also observed in close association with the cloudy white zoning lagain, see figure 18). Needles found in this form do not appear to be related to platinum inclusions.

\section{CONCLUDING THOUGHTS}

As with most other synthetic corundum, the gemological properties of the Chatham flux-grown synthetic orange and synthetic blue sapphires overlap with the properties of their natural counterparts, with the exception of inclusions. Thus, microscopic examination can provide the definitive means of identification. Among the many inclusions that are often observed, various forms of flux and platinum provide the gemologist with the most easily identifiable and diagnostic characteristics as proof of synthesis. Some inclusions, 


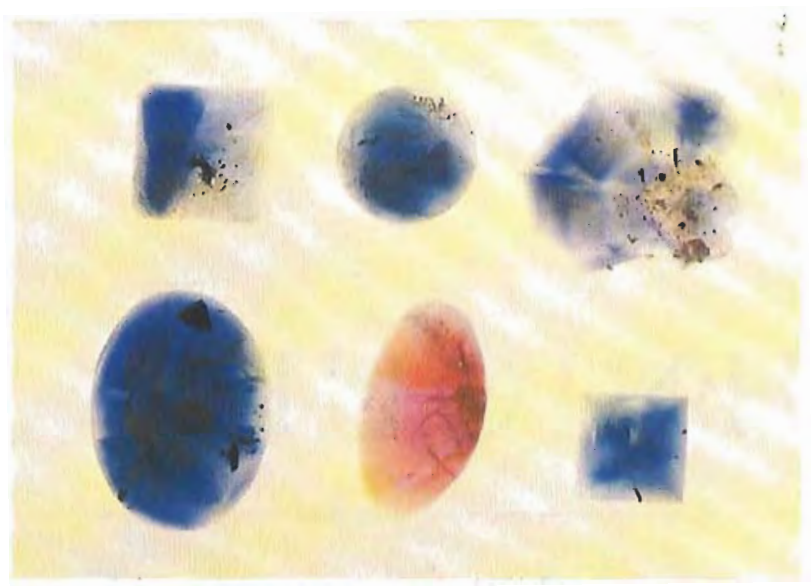

Figure 19. Immersion in methylene iodide and diffused illumination accentuate the

"hexagonal" straight and irregular color zoning in a faceted Chatham flux-grown synthetic orange sapphire and four faceted stones and one rough crystal of Chatham flux-grown synthetic blue sapphire (ranging in weight from $0.39 \mathrm{ct}$ to $1.80 \mathrm{ct}$ ).

such as straight and angular color zoning and thin, white-appearing needles could prove quite confusing if the gemologist were not aware of their possible presence.

It is difficult to determine when and if the Chatham flux-grown synthetic orange and syn-

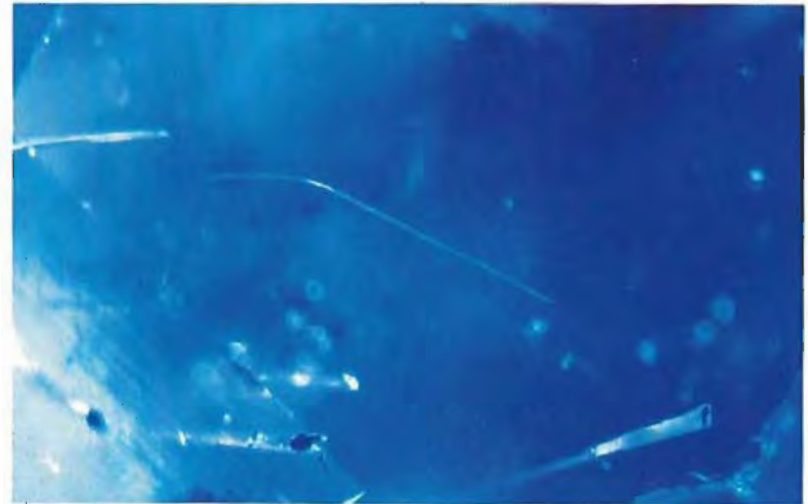

Figure 20. Thin, white-appearing needle of platinum in a Chatham flux-grown synthetic blue sapphire. Such thin platinum needles may be observed in straight or curved forms. Magnified $35 \times$.

thetic blue sapphires will become commercially available as faceted stones, since many of the crystals currently being produced are heavily included and color zoned. As changes and advances in crystal growth are made, the properties of the Chatham flux-grown synthetic orange and synthetic blue sapphire may change. Many, however, will remain essentially the same, and a complete understanding of and knowledge of these properties will be of great value to the gemologist.

\section{REFERENCES}

Abraham J. \{1982\} Heat treating corundum: the Bangkok operation. Gems \&) Gemology, Vol. 18, No. 1, pp. 79-82.

Anderson B.W., Payne C.J. (1955) The spectroscope and its application to gemmology. The Gemmologist, Vol. 25, No. 302 , pp. $158-162$.

Crowningshield R., Nassau K. (1981) The heat and diffusion treatment of natural and synthetic sapphires. Journal of Gemmology, Vol. 17, No. 8, pp. 530, 532, 533.

Kane R.E. |1979) Developments and highlights of GIA's lab in Los Angeles-synthetic rubies. Gems \&) Gemology, Vol. 16, No. 7, pp. 206-208.

Kane R.E. (1981) Gem trade lab notes-synthetic ruby. Gems (4) Gemology, Vol. 17, No. 3, pp. 163-165.

Knischka P.O., Gübelin E. (1980) Synthetische rubine mit edelsteinqualität, isometrischem habitus und holer zahl unbeschädigter kristallflächen. Zeitschrift der Deutschen Gemmologischen Gesellschaft, Vol. 29, No. 3/4, pp. 155 185. Editor's note: An abbreviated English version of this article appears in this issue of Gems $\Theta$ Gemology.
Koivula J.I. (I98 la) Brief notes on Chatham synthetic flux sapphires. Gems \&) Gemology, Vol. 16, No. 12, pp. 410, 411

Koivula J.I. (1981b) Photographing inclusions. Gems e) Gemology, Vol. 17, No. 3, p. 135.

Liddicoat R.T. Ir. (1981) Handbook of Gem Identification, 11 th ed. Gemological Institute of America, Santa Monica, CA

Nassau K. (1981) Heat treating ruby and sapphire: technical aspects. Gems $\Leftrightarrow$ Gemology, Vol. 17, No. 3, pp. 121-131

Nassau K. (1982) Colored synthetics and imitations. In International Gemological Symposium Proceedings 1982, Gemological Institute of America, Santa Monica, CA, pp. $141-150$.

Scarratt K. (1977) A study of recent Chatham synthetic ruby and synthetic blue sapphire crystals with a view to the identification of possible faceted material. Journal of Gemmology, Vol. 15, No. 7, pp. 347-353.

Webster R. (1975) Gems: Their Sources, Descriptions, and Identification, 3rd ed. Butterworth \& Co., Hamden, CT. 